\title{
Magnetic Charge Theory-The Unification of Gravity with Electricity and Magnetism
}

\author{
Keith G. Lyon \\ Haymarket, VA, USA \\ Email: klhaymarket@aol.com
}

How to cite this paper: Lyon, K.G. (2018) Magnetic Charge Theory-The Unification of Gravity with Electricity and Magnetism. Journal of Modern Physics, 9, 1381-1396. https://doi.org/10.4236/jmp.2018.97083

Received: May 8, 2018

Accepted: June 4, 2018

Published: June 7, 2018

Copyright $\odot 2018$ by author and Scientific Research Publishing Inc. This work is licensed under the Creative Commons Attribution International License (CC BY 4.0).

http://creativecommons.org/licenses/by/4.0/

\begin{abstract}
The concept of magnetic charge is further developed. The fundamental equations relating magnetic charge and magnetic fields are defined. A better photon model is developed. The ground state vibrations of particles are investigated and indicate that longitudinal waves in the ether are responsible for gravity while vortex waves can explain the Coulomb interaction. Finally, Einstein's equation for rest mass energy is derived using electromagnetic theory. A laboratory experiment is proposed to validate the theory.
\end{abstract}

\section{Keywords}

Dark Matter, Ether, Photon, Gravity, Coulomb Force, Magnetic Monopole, Nuclear Stability

\section{Introduction}

Magnetic charge was introduced to provide a mechanism where dark matter would have the properties of the ether and a transverse wave in the ether would yield the electromagnetic properties of a photon [1]. From this assumption the speed of light was derived to be proportional to the cube root of the ether density which in turn led to both space and time having the same relationship with the ether density. With space and time related to ether density and an assumption that the ether was absent in the nucleus, the Coulomb force is absent from the nucleus and with relative time going to zero, the neutron is stable. The variable speed of light also enters into the calculation of the universe's expansion where it is assumed constant; with an ether density in intergalactic space below 0.32 that here at earth, the universe's expansion will be calculated to be decelerating.

These are some very significant successes from a simple theory. The theory will be extended further in this paper, the previous photon model will be im- 
proved, and other waves in the ether will explain the transmission of the Coulomb force and gravity.

\section{Magnetic Charge versus Magnetic Monopole}

Magnetic charge and magnetic monopoles are terms that have been used interchangeably. In this paper, they have distinctly different definitions.

Magnetic monopoles have been the particles hypothesized to bring symmetry to Maxwell's equations. They have magnetic fields either emanating from them or terminating in them, depending on their polarity.

Magnetic charge is defined uniquely different. A magnetically charged particle has an associated magnetic field that is proportional to its velocity. At rest, magnetic charge has no associated magnetic field. The two definitions are illustrated in Figure 1.

Magnetic monopoles have never been detected and their existence may be impossible as a fundamental particle. Consider a stationary magnetic monopole in the presence of some moving electrical charges. The moving electrical charges are deflected by the monopole's fields and the energy to deflect these charges would come from the monopole's fields; thereby reducing the energy of the monopole. But if the energy of the monopole is changed, then the fundamental properties of the monopole are changed, which is something that should not occur for a fundamental particle. In addition, given all the electrical charges that exist, the total energy of the monopole should be quickly eliminated and thus cease to exist, if it ever existed.

The concept of magnetic monopoles provides a mechanism where magnetic fields terminate at a point and this characteristic provides symmetry to Maxwell's equations. Magnetic charge produces a similar effect; when magnetic charge moves from rest, magnetic fields originate from the resting point, and when magnetic charge stops, magnetic fields terminate at the stopping point.

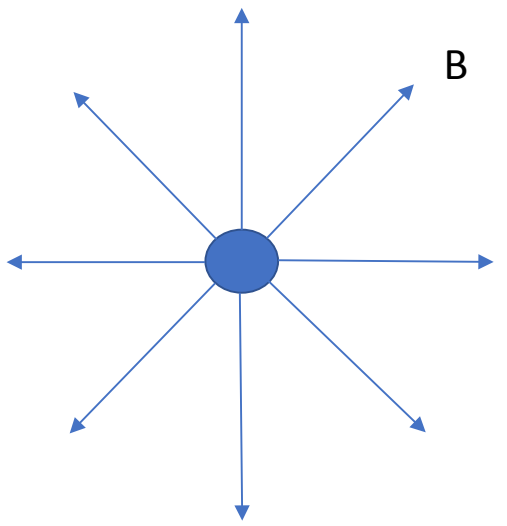

Magnetic Monopole

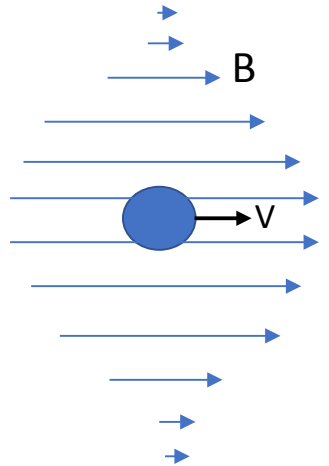

Magnetic Charge

Figure 1. Magnetic monopole/magnetic charge distinction. 
The result is that $\operatorname{div} B$ is proportional to the change in magnetic charge, $q_{m}$, hence

$$
\nabla \cdot \boldsymbol{B}=\frac{\partial q_{m}}{\partial t} .
$$

Magnetic monopoles may appear to exist as a system. A current of magnetic charges that comes to rest or are annihilated at a point would appear as a monopole with magnetic fields terminating at that point (e.g., a current of magnetic charges falling into a black hole). Likewise, magnetic charges that are accelerated from rest would appear as a monopole with magnetic fields emanating from that point (e.g., the creation of dark matter from the big bang). Thus, magnetic monopoles may effectively exist but not as fundamental particles.

\section{Theory}

The introduction of magnetic charge provides a fundamental symmetry to electricity and magnetism (EM). Currently EM has electric charge, electric fields, and magnetic fields with electric charge producing electric fields and the motion of electric charge creating magnetic fields. The addition of magnetic charge modifies EM to: electric charge and electric fields, magnetic charge and magnetic fields, the motion of magnetic charge creating magnetic fields, and the motion of electric charge moving magnetic charge and vice versa. Magnetic fields are usually encountered as closed circuits; the magnetic fields of a photon are a very notable exception and there is no moving electric charge creating this field.

In this concept, the field would be expected to be dependent on the magnitude of the magnetic charge, $q_{m}$, and its velocity, $\boldsymbol{V}$, and have a local field decreasing with range. The magnetic field from a magnetic charge is assumed to be given by the following equation:

$$
\boldsymbol{B}=q_{m} \boldsymbol{v} \mathrm{e}^{-(\rho+|z|) / \sigma}
$$

where the velocity is assumed to be in the $z$ direction, $\rho$ and $z$ are cylindrical polar coordinates centered at the magnetic charge. The distribution of the magnetic field is arbitrary, and this distribution may seem odd but, as will be shown, provides a consistent link to rest mass energy. A further discussion of this distribution and others will be discussed later in this paper. (The previous paper [1] that introduced this concept did not have this factor and only used an approximation that will be derived shortly.)

The energy in a magnetic field, $E_{B}$, in gaussian units, is [2]:

$$
E_{B}=\frac{1}{8 \pi} \int \boldsymbol{B} \cdot \boldsymbol{H} \mathrm{d} V .
$$

Inserting Equation (2) and setting $\mu=1$ yields the magnetic field energy of a magnetic charge:

$$
E_{B}=\frac{1}{16} \sigma^{3} q_{m}^{2} v^{2}
$$

The motion of the magnetic charge produces a changing magnetic field which 
in turn produces an electric field. Using Faraday's law, the electric field, $E$, is given by:

$$
\boldsymbol{E}=\frac{-q_{m} v^{2}}{c} \frac{z}{|z|} \mathrm{e}^{\frac{-(\rho+|z|)}{\sigma} \hat{\theta}}
$$

where $\hat{\theta}$ is a unit vector from cylindrical polar coordinates with the velocity in the $z$ direction, $\rho$ is the radial distance from the velocity axis, and $z$ is the distance from the particle's center. This circular electric field is localized and rotates in opposite directions leading and lagging the particle.

The electric field also contains energy, $E_{E}$, that is given by [2]:

$$
E_{E}=\frac{1}{8 \pi} \int \boldsymbol{E} \cdot \boldsymbol{D} \mathrm{d} V \text {. }
$$

Inserting Equation (5) into Equation (6) and setting $\varepsilon=1$ yields an electric field energy:

$$
E_{E}=\frac{1}{16} \sigma^{3} q_{m}^{2} \frac{v^{4}}{c^{2}} .
$$

Inserting Equation (4) yields the electric field energy in terms of the magnetic field energy:

$$
E_{E}=E_{B}\left(\frac{v^{2}}{c^{2}}\right) .
$$

The electric field energy is insignificant at low velocities $(v \ll c)$ compared to the magnetic field energy. At high velocities the electric field energy becomes more significant and approaches the magnetic field energy as $v$ approaches $c$.

The total field energy, $E_{E+B}$, is obtained by adding Equations ((4) and (7)), and is given by:

$$
E_{E+B}=\frac{1}{16}\left[1+\frac{v^{2}}{c^{2}}\right] \sigma^{3} q_{m}^{2} v^{2}
$$

At low velocities, the energy of a magnetic charge is proportional to $v^{2}$, just as kinetic energy, which is the first clue to connecting magnetic charge with mass.

For later in this paper, a useful calculation is the magnetic field associated with a uniform flow of magnetic charges. Assuming a magnetic charge particle density, $d$, with each charge, $q_{m}$, yields an average magnetic field of:

$$
\boldsymbol{B}=q_{m} \boldsymbol{v}\left(d \sigma^{3}\right) \text {. }
$$

The overall magnetic field is a factor $\left(d \sigma^{3}\right)$ times the peak magnetic field of a single magnetic charge. This provides a useful approximation where magnetic field variations are large compared to magnetic charge separations and $\sigma$, which, will be shown later, are both very small.

The forces on a magnetic charge are somewhat different than its electrical counterparts. An electric charge in an electric filed is constantly accelerated and can attain high velocities. A magnetic charge when released into a magnetic field is immediately accelerated but the acceleration slows as the charge's magnetic 
field blends with the external magnetic field, much like a drop of water released into a flowing river.

\section{Ether}

The conclusion that the ether exists conflicts with the standard teachings that the photon is a pure electromagnetic wave. A reexamination of the logic that led to that conclusion would seem to be required.

Jackson [2] presents a rational explanation of how this conclusion was reached by citing the results of three experiments: the observation of the aberration of star positions during the year, Fizeau's experiment on the velocity of light in moving fluids, and the Michelson-Morley experiment to detect motion through the ether. These experiments provided contradictory characteristics of the ether and a single ether concept could not be identified that was consistent with all the observations.

The results of these experiments were explained by Einstein's theory of special relativity. Special relativity is a very general theory that makes no assumptions with respect to an ether and its success also has nothing to do with an ether. The more appropriate conclusion of the ether experiments is that classical physics cannot be used to characterize the ether; it is a phenomenon in relativistic physics. The confusing results are the outcome of trying to explain a relativistic problem with classical mechanics. All classical analyses of these experiments involved the simple addition or subtraction of a velocity with the speed of light, which we now know cannot be done with relativistic velocities. At the time of the ether debate, special relativity was not a staple in physics as it is now. Hence, the conclusion that the ether did not exist is understandable and the debate not being revisited is a result of cognitive dissonance. Therefore, the existence of an ether would still be a possibility.

If this theory is correct, then the ether would consist of magnetic charges. To have avoided detection, the particles of the ether would have a very low mass density, so low that it is only now being detected in astronomical quantities as dark matter. The detection of dark matter may very well turn out to be the detection of the ether.

\section{Transverse Photon}

The term transverse photon, or t-photon, is introduced to signify a photon created by a transverse wave in the ether (and to differentiate from other types of photons that are going to be introduced). The t-photon is the same as the historical photon.

Developing a model for the $\mathrm{t}$-photon is possible, in fact many models can be hypothesized. Any model can be used to calculate the energy in the magnetic and electric fields, and these energies can be compared to the known energy of a t-photon, $h f$. In addition, any plausible model would need to be consistent with the well-known particle-wave duality of the t-photon. 
Several models were considered starting with a simple string (not to be confused with string theory) of vibrating dark matter. In the case of the single string, the maximum velocity of dark matter exceeded the speed of light by several orders of magnitude. To obtain a model that had reasonable values for magnetic charge and dark-matter velocities that do not exceed the speed of light, the t-photon needed to be rather large compared to the wavelength. This also provides consistency with diffraction from a dual slit when the slits are widely separated.

The model that appears to be the most characteristic of the observed photon properties is based on a single string of dark matter at its center that, as it vibrates, affects their nearest neighbors which in turn affects their nearest neighbors, etc. This center string has the largest vibration amplitude and the amplitudes of its neighbors are smaller at larger distances. In addition, at larger t-photon energies, the peak amplitude increases while its breadth is reduced. (This aspect is a departure from the model previously assumed [1] which was the result of forcing a photon to be scaled equally in all three dimensions. The model presented here is a much better fit to observed phenomenon.) Assuming that amplitudes are gaussian in all three directions, leads to the following equation for the displacement of dark matter, $d$, from its initial static location, $(x, y, z)$, at an instant in time with the center of the t-photon at the origin:

$$
d(x, y, z)=A_{0} f \sin (k x) \mathrm{e}^{-x^{2} / \sigma_{x}^{2} \lambda^{2}} \mathrm{e}^{-y^{2} / \sigma_{y}^{2} \lambda^{2}} \mathrm{e}^{-z^{2} / \sigma_{z}^{2} \lambda^{2}} .
$$

$A_{0} f$ is the peak displacement at the center of the t-photon, $k$ is the wavenumber $(2 \pi / \lambda), \sigma_{i}$ is the standard deviation in the $i$ direction, and $\lambda$ is the wavelength. $\sigma_{i}$ is a unitless number since the size of the t-photon should logically scale with wavelength. This equation assumes the photon propagation is in the $x$ direction and the $x-y$ plane is the vibration plane.

The first order assumption to the nature of a vibration would be a simple harmonic oscillator, therefore, the velocity of the dark-matter particle, $v$, is given by:

$$
v(x, y, z)=A_{0} 2 \pi f^{2} \cos (k x) \mathrm{e}^{-x^{2} / \sigma_{x}^{2} \lambda^{2}} \mathrm{e}^{-y^{2} / \sigma_{y}^{2} \lambda^{2}} \mathrm{e}^{-z^{2} / \sigma_{z}^{2} \lambda^{2}} .
$$

Since the total electromagnetic energy, $E_{B+E}$ of the t-photon is twice the magnetic field energy given by Equation (3), the total photon energy is given by:

$$
E_{B+E}=\frac{1}{4 \pi} \int \boldsymbol{B} \cdot \boldsymbol{H} \mathrm{d} V .
$$

Substituting the equation for velocity, Equation (12), into the equation for the approximate magnetic field, Equation (10), and solving the integral provides the following relationship in terms of the model parameters:

$$
E_{B+E}=\frac{1}{16} \sqrt{\pi} \pi^{2} \mu q_{m}^{2} d_{d m}^{2} \sigma^{6} A_{0}^{2} c^{3} \sigma_{x} \sigma_{y} \sigma_{z} f .
$$

This result indicates that the electromagnetic energy of the t-photon is pro- 
portional to $f$ as expected ${ }^{1}$.

The t-photon is an obvious quantum state as it exists only with an energy $h f$ and does not transform to another state by losing energy. Consider the creation of a t-photon from the electron transition in an atom; the atom is about $10^{-8} \mathrm{~cm}$ in size yet creates t-photons with wavelengths many orders of magnitude larger. If the creation and collapse of the quantum state are assumed to be mirror images of each other, then the t-photon starts at a point and collapses to a point, yielding the particle-like nature of the t-photon. Between creation and collapse, the $\mathrm{t}$-photon exists as a localized transverse wave providing the wave-like properties. At collapse, its energy and momentum converge to a point and appear like a particle.

The t-photon also has kinetic energy given by:

$$
E_{K}=\sum_{i} \frac{m_{i} v_{i}^{2}}{2}=\frac{1}{2} \int m_{d m} d_{d m} v^{2} \mathrm{~d} V
$$

where the sum is over all dark-matter particles in the t-photon, $m_{i}$ is the mass of an individual dark-matter particle, and $v_{i}$ is the velocity of that particle given by Equation (12). Assuming each dark-matter particle has the same mass, $m_{d m}$, and a particle density $d_{d m}$, this becomes:

$$
E_{K}=\frac{1}{2} \sqrt{\frac{\pi}{2}} \pi^{3} m_{d m} d_{d m} A_{0}^{2} c^{3} \sigma_{x} \sigma_{y} \sigma_{z} f .
$$

Both the kinetic energy and electromagnetic field energy of a t-photon are proportional to $f$. This is the second clue that connects magnetic charge with mass.

Overall, this analysis indicates that:

- the t-photon is considerably larger than the wavelength,

- the t-photon is the same as a phonon, but is a vibration in dark matter instead of normal matter,

- the t-photon has an electromagnetic energy and kinetic energy that are both proportional to frequency, and

- the t-photon is a quantum state whose creation and collapse provide the particle-like characteristics, while the steady state properties provide the wave-like characteristics.

\section{Dark-Matter Properties}

The previous paper calculated bounds on the characteristics of dark-matter particles. An old value for the dark matter density was used. If a newer value of $8 \times$ $10^{-25} \mathrm{~g} \mathrm{~cm}^{-3}$ is assumed, the upper bound for the mass of a dark matter particle would be about $2 \times 10^{-79} \mathrm{~g}$. The other properties remain the same, namely, the upper limit on the spacing between dark-matter particles is about $6.2 \times 10^{-19} \mathrm{~cm}$

\footnotetext{
${ }^{1}$ In my initial paper, I assumed a photon model that did not scale the amplitude with frequency in a manner parallel with plane wave models usually used for photons. The results were that electromagnetic energy was proportional to wavelength and I concluded that the energy of the photon was kinetic, just as a phonon. Further work, as shown later in this paper, shows that this could not be the case if a connection to gravity was to exist.
} 
and a dark-matter particle density greater than $4.2 \times 10^{54} \mathrm{~cm}^{-3}$.

\section{Photon Generation}

T-photons are generated by many means. In an atom the transition of an electron from a higher energy state to a lower state generated a t-photon that was described above to explain the particle-wave duality. Another means of producing photons are antennas. The simple dipole antenna provides an example where t-photons are gradually produced in their near-final form, whereas the electron transition produced a quantum of energy that grew to the final t-photon form and then later collapsed to a quantum of energy at a point.

In the dipole antenna situation, t-photons are formed as the electric field in the antenna oscillates. This oscillating electric field creates an oscillating magnetic field around the antenna that propagates outwardly from the antenna. From the magnetic field equation, Equation (2), the instant the magnetic field is created next to the dipole antenna, the ether starts to move and in so doing creates magnetic field further outside the antenna, which then affects additional ether, etc. This nearest neighbor interaction creates a wave, which in this case, is in almost the final form of a t-photon. If the antenna were only energized for one period, waves that are one wavelength long would be generated that would grow to the final t-photon size, considerably larger than a single wavelength, hence the use of the term near-final form. While this is a somewhat useful image of t-photon formation, the insight is useful to see that any movement in the ether can create waves, in this case these waves coalesce into the quantized $\mathrm{t}$-photons. Other movements may or may not create quantized entities that can propagate at the speed of light as does a t-photon, but some may.

From quantum mechanics, the ground state of the simple harmonic oscillator has a nonzero energy. For a three-dimensional harmonic oscillator, the ground state energy is $3 h f / 2$. If this applies to elementary particles, it implies that all particles are vibrating even at absolute zero. Since these particles are in the ether, these vibrations would create waves as they interact with the ether, just as a vibrating stick in water would create waves. Clearly, a vibrating proton or electron would also disturb the ether due to their electric charge. A neutron could also create a disturbance, if only by collisions with the ether. If a particle has a magnetic charge, the magnetic field from the vibration would also cause a wave. If the ground state of a particle has an energy equal to its rest mass energy, $m c^{2}$, then a proton or neutron would have a frequency of about $10^{23} \mathrm{~s}^{-1}$. At this frequency, the amplitude would need to be quite small $\left(<10^{-14} \mathrm{~cm}\right)$ to avoid peak velocities that exceed the speed of light. However, this peak amplitude is still large compared to the average spacing between dark-matter particles that was previously estimated $\left(<6 \times 10^{-19} \mathrm{~cm}\right)$ [1]. (In the following sections, the assumption that the fundamental vibration frequency of a particle will be shown to not be proportional to the rest mass but the concept of a particle's vibration being very large is consistent with this theory.) The next two sections will discuss the 
two most interesting waves that are associated with particle vibrations.

\section{Gaussian Photon}

Consider the fundamental vibration of a magnetically charged, but not electrically charged, particle. Since the separation distance between dark-matter particles is much smaller than the size of the fundamental particles, the motion will create magnetic fields both by physically moving the dark matter, creating a magnetic field in the velocity direction, as well as, creating a magnetic field from the motion of the magnetic charge, Equation (2). While the physical motion creates an approximately spherical longitudinal wave through the dark matter, the magnetic field produced by the moving magnetic charge creates a magnetic field and a larger, more significant wave.

The predominant field energy distribution from this motion is gaussian-shaped, Equation (2), and the resulting longitudinal wave is assumed to also be gaussian, which forms the definition of the gaussian photon, or g-photon. The direction of this wave depends on the polarity of the magnetic charge; if the polarity of the charge is the same as dark matter then the wave propagates in the velocity direction; if the polarity is opposite that of dark matter, the wave propagates in the opposite direction.

If this vibration was one dimensional, then each half cycle would create a g-photon and with multiple cycles, g-photons would alternately be transmitted in opposite directions. The vibrations of a particle are three dimensional, so g-photons are transmitted in random directions forming a spherical distribution. The vibrating, magnetically charged particle creates g-photons with each g-photon having a magnetic field proportional to the magnetic charge of the particle and the peak velocity of the vibrations. This field of g-photons is spherically distributed, so their density is inversely proportional to the range squared. The rate at which these g-photons are generated is proportional to the fundamental vibration frequency. Hence, the average field strength, $\langle B\rangle$, is proportional to the magnetic charge, $q_{m}$, times the vibration frequency, $f_{r}$, times the peak velocity, $v_{p}$, divided by the range squared:

$$
\langle B\rangle \propto \frac{q_{m} f_{v} v_{p}}{r^{2}} .
$$

\section{Vortex Photon}

Consider the fundamental oscillating vibration of an electrically charged particle. Since it is electrically charged, each vibration creates an electric current, which creates a magnetic field about its motion, i.e., a circular motion of the ether about the direction of that vibration. While the magnetic field about the particle is caused by the particle's motion, the result is to create a wave with similar properties, specifically a vortex about the particle's motion that propagates in the direction of the particles motion, a vortex photon or v-photon. The vortex rotation direction depends on the polarity of the electric charge, thus there are two 
types of vortex photons, one for positive charges, $\mathrm{v}^{+}$-photons, and the other for negative charges, $\mathrm{v}^{-}$-photons. Since the particle's vibrations are random, vortices are sent in all directions with each vortex representing a single half vibration from the particle that may be quantized, hence the term vortex photon. Also, at the rest-mass assumed frequencies for the vibration, the v-photon is very small having been generated by the particle's motion of $<10^{-14} \mathrm{~cm}$. Thus, for a proton, there are $10^{+23}$ tiny v-photons being emitted every second, if vibrating at the rest mass energy.

The rotation direction for the v-photon depends upon the polarity of the charge and, from Ampere's Law, produces an electric field whose direction depends on the polarity of the particle's electric charge. For a positive charge, the electric field of the $\mathrm{v}^{+}$-photon would be in the velocity direction and vice versa

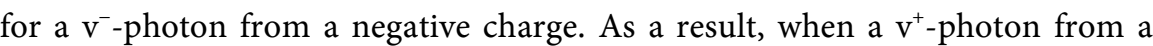
positive charge interacts with another positive electrical charge, the electric field repels the second positive charge; whereas, if the $\mathrm{v}^{+}$-photon interacts with a negative electric charge, it attracts this charge. Thus, the v-photon provides a pulse of electric field that exerts the Coulomb force. This is a significant advantage over a virtual photon concept since the communication occurs at the speed of light, matching the observed reaction delay.

An interesting result is obtained by examining the forces with this model. The magnetic fields associated with the v-photon are proportional to the vibrating electric charge's charge, $q_{e}$, and peak velocity, $v_{p}$. The electric field of the v-photon is determined by Ampere's Law and the result is that the electric field is proportional to the electric charge and the amplitude of the oscillation, $A$. The $\mathrm{v}$-photons emitted by the charge are spherically distributed and emitted at a rate proportional to the frequency of the vibration, $f_{v}$. Thus, the average electric field, $\langle E\rangle$, at a distant point is proportional to the electric charge times the oscillation amplitude times the frequency and inversely proportional to the distance squared:

$$
\langle E\rangle \propto \frac{A q_{e} f_{v}}{r^{2}} \propto \frac{q_{e} v_{p}}{r^{2}} .
$$

The frequency times the amplitude for a simple harmonic oscillator is proportional to the peak velocity, $v_{p}$, of the vibration. If this peak velocity of the vibration is a constant, then the electric field of this distribution of v-photons would be the same as that of the classical electrical field from a charged particle. The result that all matter vibrates with the same peak velocity is interesting, especially since the only velocity that is fundamentally constant is that of light.

\section{Gravity}

Twice in this paper there were clues that mass and magnetic charge are related. When the kinetic energy of a magnetically charged particle was compared to the electromagnetic field energy of the same particle, they were found to both be proportional to $v^{2}$. The energies could be equal if the mass was proportional to 
the square of the magnetic charge. For the photon, a similar conclusion relating mass and magnetic charge was observed when comparing the photon's field and kinetic energies. The g-photon with specific conditions provides a third clue.

Consider that the vibrating, magnetically charged particle described above has a polarity opposite to that of the dark matter. When the propagated wave from that particle interacts with a particle of the same polarity (opposite to dark matter), the intercepted particle will experience an attractive force. If all matter were magnetically charged with a polarity opposite to dark matter, then these vibrating, magnetically charged particles would all be attracted to each other in a manner like gravity.

The average magnetic field associated with a vibrating magnetic charge was determined above to be proportional to the magnetic charge times the vibration frequency times the peak velocity divided by the range squared, Equation (17). The previous section determined that if the v-photon is responsible for the Coulomb force, the peak velocity of all vibrations is a constant, thus the field associated with a vibrating magnetic charge is reduced to being proportional to the magnetic charge times the frequency divided by the range squared. If frequency were proportional to magnetic charge, then this field would be proportional to the magnetic charge squared divided by the range squared, which would be the same as the gravitational potential if mass were proportional to the magnetic charge squared, as seen in the first two clues.

Another observation that supports the conclusion that gravity is an electromagnetic interaction, is that gravity reacts at the speed of light. This would imply that the ether is responsible for the transmission of the force.

The correlation of mass with magnetic charge squared requires that all matter is vibrating with the same peak velocity and the vibration frequency is proportional to the magnetic charge. Both assumptions are perplexing. It would be logical if the peak velocity was the speed of light, but any other value would be illogical. On the other hand, there does not seem to be a logical reason why the frequency is proportional to the magnetic charge (or the square root of the mass).

\section{Magnetic Fields from Moving Matter}

The assumption that all matter has magnetic charge can provide the parallel to gravity as described above but leads to the question of why a magnetic field has not been detected when any matter is in motion if the matter is magnetically charged. The equations that provided the first two clues in connecting mass and magnetic charge also provide the explanation for not detecting a magnetic field.

Equating the t-photon's field and kinetic energies, Equations ((14) and (16)), provides the following relationship for the mass of a dark-matter particle:

$$
m_{d m}=\frac{\sqrt{2}}{8 \pi} d_{d m} \sigma^{6} q_{d m}^{2}
$$


Similarly, setting the magnetic charge's field energy at low velocity, Equation (9), equal to the kinetic energy, $m v^{2} / 2$, yields the general relationship between mass and magnetic charge:

$$
m=\frac{1}{8} \sigma^{3} q_{m}^{2}
$$

Equating these equations for dark matter results in:

$$
d_{d m} \sigma^{3}=\frac{\pi}{\sqrt{2}} .
$$

This implies that the separation between dark-matter particles is approximately $0.766 \sigma$. This separation was previously estimated to be $<6.2 \times 10^{-19} \mathrm{~cm} \mathrm{[1]}$ which means that $\sigma<4.8 \times 10^{-19} \mathrm{~cm}$.

A $\sigma$ this small indicates that the field from a magnetic charge is extremely limited in extent. At the Bohr radius, the field is reduced by about $10^{+9}$ orders of magnitude. Thus, the motion of a nucleus would produce a field that could not be detected outside the atomic shell. Even at relativistic velocities and a heavy nucleus, the magnetic field would not increase to a detectable level at the edge of the atom, and much less at visible distances. Even multiplying by Avogadro's number would result in no detectable field.

Equation (21) also implies that $\sigma$ is defined by the dark-matter density, which at first, seems odd. However, for the laws of physics to be invariant, fundamentals need to scale in every reference frame. In this case, the separation of dark-matter particles is equal to $0.766 \sigma$ in all frames of reference, which means the magnetic field strength of a dark-matter particle is always about $46 \%$ of its peak value at its nearest neighbor.

\section{Rest Energy}

The ground state vibration provides a means to calculate the rest energy of a magnetic charge. Assuming that the ground state vibration is a simple harmonic oscillator, the total energy is equal to the peak energy at the maximum velocity. The above results indicated that all particles have the same peak velocity, and the only velocity that is a natural constant is that of light. As a result, inserting the speed of light into Equation (9) gives the following:

$$
E_{E+B}=\frac{1}{8} \sigma^{3} q_{m}{ }^{2} c^{2} .
$$

Substituting the general relationship between mass and magnetic charge, Equation (20), results in:

$$
E_{E+B}=m c^{2} .
$$

When Equation (2) was introduced, an exponential decay was assumed. While logic dictates that the field should decay with range, there are no obvious choices. A more general expression for Equation (2) is:

$$
\boldsymbol{B}=q_{m} \boldsymbol{v} f(\boldsymbol{r}),
$$

where $f(r)$ is a general decay function. In preliminary analysis leading to this pa- 
per, a gaussian decay was assumed but Equation (23) was smaller (0.64 $\left.m c^{2}\right)$. Other decays were also studied but resulted in even lower values for Equation (23). Further analysis indicated that any decay function, $f(r)$, satisfying the following will produce Equation (23):

$$
\frac{\partial f}{\partial \rho}=\frac{\partial f}{\partial z} .
$$

The decay function selected in Equation (2) was the simplest function meeting this constraint, but it may not be unique.

Deriving the rest mass energy, Equation (23), from the assumption of magnetic charge, Maxwell's equations, and no special relativity equations is the final clue that mass and magnetic charge are related.

\section{Units}

Units in EM have always been confusing. For example, inspection of Faraday's Law in gaussian units [2],

$$
\nabla \times \boldsymbol{E}+\frac{1}{c} \frac{\partial \boldsymbol{B}}{\partial t}=0
$$

would imply that $E$ and $B$ should have the same units and this theory would then imply that there exists some relationship between electric and magnetic charge. Yet, $E$ has the units of statcoulombs $\mathrm{cm}^{-2}\left(\mathrm{sc} \mathrm{cm}^{-2}\right)$ and $B$ has the units of gauss. Equation (2) implies that magnetic field should have units of magnetic charge times velocity, which would further complicate existing EM units. Instead, all units can be defined by time, distance, and electric charge. Magnetic field can have units $\mathrm{sc} \mathrm{cm}^{-2}$, if magnetic charge has units sc sec $\mathrm{cm}^{-3}$. Similarly, other units can be defined in terms of time, distance, and electric charge and are given in Table 1. (Magnetic charge units or field units could have been the basis (instead of electric charge) but would have required the definition of a new unit.) While this reduces the complexity of EM units, the key result is that all units can be defined from three units: length $(\mathrm{cm})$, time $(\mathrm{sec})$, and electric charge (sc).

Table 1. Units.

\begin{tabular}{cc}
\hline DISTANCE & $\mathrm{CM}$ \\
TIME & $\mathrm{sec}$ \\
ELECTRIC CHARGE & statcoulomb (sc) \\
ELECTRIC FIELD & $\mathrm{sc} \mathrm{cm}^{-2}$ \\
MAGNETIC CHARGE & $\mathrm{sc} \mathrm{sec} \mathrm{cm}$ \\
MAGNETIC FIELD & $\mathrm{sc} \mathrm{cm}^{-2}$ \\
ENERGY & $\mathrm{sc}^{2} \mathrm{~cm}^{-1}$ \\
FORCE & $\mathrm{sc}^{2} \mathrm{~cm}^{-2}$ \\
MASS & $\mathrm{sc}^{2} \mathrm{sec}^{2} \mathrm{~cm}^{-3}$ \\
\hline
\end{tabular}




\section{Nuclear Stability}

The previous paper [1] pointed out that the lack of dark matter in the nucleus leads to an inability to transmit the Coulomb force between protons and the stability of the neutron. (A corollary to this is that as two protons approach each other, the repulsive force increases until they are so close that no dark matter exists between them, at which time, the repulsive force disappears.) The lack of dark matter in the nucleus also means that g-photons cannot be transmitted between nucleons. Therefore, the gravitational attraction between nucleons also does not exist inside the nucleus.

The lack of both the Coulomb and gravitational forces leaves the question of how EM affects nuclear cohesion. The particle vibrations that generated v-photons and g-photons, first created magnetic fields that accelerated dark matter which created the photons. The magnetic fields from vibrating nucleons still exist in the nucleus. If these vibrations become coupled and synchronized, they might create a bound state. Consider Deuterium, if the proton and neutron are synchronized (i.e., they are in a dance with one ahead of the other in the velocity direction), their fields would be aligned, and the nucleons would be attracted like magnets. The particles would stay synchronized, if one continues to lead the other in their random vibrations. This is a trivial example for a complex multibody problem with no assurance that there is a general solution, but the possibility exists; however, the analysis is beyond the scope of this paper.

\section{Impacts to Astrophysics}

The variability of the speed of light across the universe further complicates the determination of large distances. Calculations based on luminosity are unaffected. However, calculations that depend on knowing the speed of light require knowledge of the variations of the dark-matter density along the light's path. The reduced speed of light in intergalactic space will significantly reduce the estimates for the size of the universe and its expansion rate. The previous paper used calculations from Tangherlini [3] to calculate a dark-matter density in intergalactic space of $32 \%$ of the dark-matter density at Earth to achieve a zero-expansion rate, and a more logical value will be less than $32 \%$ to imply a decelerating expansion. Tangherlini also points out that the index of refraction of dark matter needed for this result would imply a divergence of light passing a massive object that is opposite to the effect of gravitational lensing. The analogy with refraction has a fundamental flaw in that refraction is the interaction of a photon with normal matter, while gravitational lensing is a result of the photon interaction with a gradient in space-time. In essence, a photon passing a massive object must slow on the photon's side near the object so that the photon state can remain coherent with the far side given the differences in their reference frames.

This theory provides a new possible explanation to accretion discs. Accretion discs are characterized by a circulating disc of material spiraling into a large 
central mass and mass being ejected from the central mass normal to the disc's plane. Assuming the spiraling mass is constituted of dark matter and normal matter in a ratio similar to the rest of space, i.e., more dark matter than normal matter, a magnetic field will be generated in the direction of the mass flow and proportional to its velocity. This has two key effects. First the circular magnetic field produces, via Ampere's Law, an electric field at the center of the disc and perpendicular to the disc's plane. Given the astronomical amount of dark matter generating these fields and the high velocities near the central mass, the electric field could be significant, significant enough to ionize atoms and eject the charged particles at high energies normal to the plane. The second effect is that the predominant dark matter creates a magnetic field that slows the normal matter since it has the opposite magnetic polarity. This effect reduces the energy of the normal matter, causing the matter to slow and flow into the center faster than orbital drag would predict, where it is ionized and ejected. This analysis implies that one jet of mass from the center is positively charged and the other jet negatively charged (electrically).

\section{Experimental Verification}

Superconductors have a very unique place in EM. Magnetic fields do not penetrate a superconductor, which in this theory would imply that there are no dark-matter currents in the superconductor. However, dark matter must exist inside the superconductor to transmit the Coulomb force between nuclei and electrons. Thus, the dark matter can vibrate but must exist in some bound state. This may provide a means to create a volume of space with a variable dark-matter density as suggested previously [1].

Suppose one creates a hollow cylinder with an integral cap on one end made of superconducting material. This can must be made of a single piece of material without joints so that the only dark-matter current into or out of the can is through the open end. The inside bottom of the can should be polished to provide a good reflector of light. By inducing a current around the opening of the can, a magnetic field can be created that depending on the current direction, would either fill the can with dark matter or pump the dark matter out of the can. If pulses of light were reflected off the bottom of the can back to a receiver outside the can, the transmit time could be determined as the dark-matter density changes. Changes in this transit time would indicate a change in the speed of light and a validation of this theory.

\section{Summary}

The concept of magnetic charge led to the conclusion that all matter has magnetic charge with normal matter and dark matter having opposite polarities. The evolution of the concept indicates that photons are a result of a transverse wave in a uniform distribution of magnetically charged particles, hence the conclusion that dark matter is the ether. Additionally, other waves are compatible with the 
propagation of the Coulomb and gravitational forces. Thus, waves in dark matter provide a mechanism for energy transfer ( $\mathrm{t}$-photons), transmission of the Coulomb force (v-photons), and gravity (g-photons). Fundamental to these results is that all matter has a ground state vibration, which led to the conclusion that all matter vibrations have the same peak velocity, and the vibration frequency is proportional to its magnetic charge. A variable dark-matter density across the universe leads to a variation in the speed of light and hence, a variation in space-time. The variation in the speed of light leads to the possibility that the universe is decelerating when appearing as though the universe's expansion is accelerating. In the tightly packed nucleus, the lack of a Coulomb force and the neutron stability can be explained by an absence of dark matter. The most intriguing result of the concept is that mass is a calculated property of a particle and that the fundamental properties of a particle are electric and magnetic charges. Finally, the rest energy of a magnetically charged particle is shown to be equivalent to $m c^{2}$.

The existence of dark matter is fundamental to the existence of space and time as we know it. Without dark matter, our universe would not exist, and magnetic charge is basic to the fundamental laws of physics.

\section{References}

[1] Lyon, K.G. (2016) Journal of Modern Physics, 7, 920-927. https://doi.org/10.4236/jmp.2016.79084

[2] Jackson, J.D. (1962) Classical Electrodynamics. John Wiley \& Sons, Inc., New York.

[3] Tangherlini, F.R. (2015) Journal of Modern Physics, 6, 1360-1370. https://doi.org/10.4236/jmp.2015.69141 